PROCEEDINGS OF THE

AMERICAN MATHEMATICAL SOCIETY

Volume 129, Number 3, Pages $765-770$

S 0002-9939(00)05579-9

Article electronically published on August 30, 2000

\title{
ON THE TEICHMÜLLER THEOREM AND THE HEIGHTS THEOREM FOR QUADRATIC DIFFERENTIALS
}

\author{
SHENGJIAN WU
}

(Communicated by Albert Baernstein II)

\begin{abstract}
By using the Marden-Strebel heights theorem for quadratic differentials, we provide a concrete method for finding the Teichmüller differential associated with the Teichmüller mapping between compact or finitely punctured Riemann surfaces.
\end{abstract}

\section{INTRODUCTION}

Let $R$ be a compact or finitely punctured Riemann surface of hyperbolic type, that is, the universal cover of $R$ is the unit disc. For a Riemann surface $R$, the vector space of all holomorphic quadratic differentials $\varphi$ with finite norm

$$
\|\varphi\|=\iint_{R}|\varphi| d x d y
$$

will be denoted by $Q(R)$ and the unit sphere in $Q(R)$ will be denoted by $Q_{0}(R)$.

Let $g: R \rightarrow S$ be an orientation preserving homeomorphism. The famous Teichmüller theorem tells us that there is a Teichmüller mapping in the homotopy class of $g$, that is, there is a quasiconformal mapping $f: R \rightarrow S_{0}$ in the homotopy class of $g$ such that the complex dilatation $\mu_{f}$ of $f$ has the following form:

$$
\mu_{f}=k \frac{\bar{\varphi}}{|\varphi|}
$$

where $k(0 \leq k<1)$ is a constant and $\varphi \in Q_{0}(R)$. In the following, we say that $\varphi$ is associated with the Teichmüller mapping $f$.

In this note we will consider the following problem: Given a homeomorphism $g: R \rightarrow S$, find the quadratic differential $\varphi \in Q_{0}(R)$ such that it is associated with the Teichmüller mapping $f: R \rightarrow S$ which is homotopic to $g$.

For this purpose, we note that, for the Teichmüller mapping $f$, if we denote its complex dilatation by $\mu_{f}$, then we have the following well-known result which is due to Hamilton, Krushkal, Reich and Strebel.

Received by the editors March 6, 1999 and, in revised form, May 6, 1999.

2000 Mathematics Subject Classification. Primary 30F10, 30F60.

Key words and phrases. Quasiconformal mapping, Teichmüller differential, Hamilton sequence, quadratic differentials.

This work was supported by the SRF for ROCS, SEM and the NSF of China.

(C)2000 American Mathematical Society 
Theorem A ([1]). Let $f: R \rightarrow S$ be a quasiconformal mapping. Then $f$ is a Teichmüller mapping if and only if

$$
\sup _{\varphi \in Q_{0}(R)}\left\{\operatorname{Re} \iint_{R} \mu_{f} \varphi\right\}=\left\|\mu_{f}\right\|_{\infty} .
$$

A sequence $\left\{\varphi_{n}\right\}_{1}^{\infty} \subset Q_{0}(R)$ such that the above supremum is attained is called a Hamilton sequence for $f$ or for $\mu_{f}$. Since $R$ is conformal finite type, any Hamilton sequence $\varphi_{n}$ must be convergent to $\varphi_{0}$ which is associated with the Teichmüller mapping $f$.

Therefore our problem is equivalent to how to find a Hamilton sequence. To our knowledge, it seems that there is no concrete way known so far.

On the other hand, in some mathematical problems, iteration often leads to interesting results. In this note, we shall, by considering some kinds of iteration of the Marden-Strebel mapping by heights (cf. $\S 2$ ), provide a concrete way for finding a Hamilton sequences.

\section{Some KNOWN Results on the MAPPING BY Heights}

Let $\varphi=\varphi(z) d z^{2}$ be a quadratic differential in $Q_{0}(R)$. Then $\varphi$ defines two invariants, the area element $d A=|\varphi| d x d y$ and the line element $d s=\sqrt{|\varphi(z)|} d z \mid$. The $\varphi$-length of an arc $\gamma$ in $R$ is $\int_{\gamma} d s$, and the height of $\gamma$ with respect to $\varphi$ is $h_{\varphi}(\gamma)=\int_{\gamma}|\operatorname{Im} \sqrt{\varphi(z)} d z|$. For any $\varphi \in Q(R)(\varphi \not \equiv 0)$ and for any piecewise smooth closed loop $\gamma$ on $R$ which is not homotopic to a point one can define the height of its free homotopy class $[\gamma]$ by

$$
h_{\varphi}(\gamma):=\inf _{\gamma^{\prime} \sim \gamma} \int_{\gamma^{\prime}}|\operatorname{Im} \sqrt{\varphi(z)} d z|>0 .
$$

In [2], Marden and Strebel introduced the mapping by heights $g_{h}$ that associates with each differential $\varphi \in Q(R)$ a differential $\psi \in Q(S)$ which is uniquely determined by the property that for any $\gamma$ on $R$

$$
h_{\psi}(g(\gamma))=h_{\varphi}(\gamma)
$$

Define the normed mapping by heights, for $\varphi \in Q_{0}(R)$, by

$$
g_{\#}(\varphi)=\frac{g_{h}(\varphi)}{\left\|g_{h}(\varphi)\right\|}
$$

These authors proved that $g_{\#}$ is a homeomorphism from $Q_{0}(R)$ to $Q_{0}(S)$ and, by analyzing $g_{\#}$, gave a geometric proof of the Teichmüller theorem (cf. [2] and [3]). Furthermore they proved the following result.

Theorem B (3]). A necessary and sufficient condition for $\varphi \in Q_{0}(R)$ to be associated with a Teichmüller mapping $f: R \rightarrow S$ homotopic to $g$ is that

$$
g_{\#}(-\varphi)=-g_{\#}(\varphi)
$$

and $\left\|g_{h}(\varphi)\right\| \geq 1$.

Note that the condition that $\left\|g_{h}(\varphi)\right\| \geq 1$ is not included in the original theorem in [3]. On the other hand, it is easy to see from the arguments in [3] that the above result, which will be suited to our applications, can be deduced. In fact, if $\varphi \in Q_{0}(R)$ is the Teichmüller differential associated with $f$, then $\left\|g_{h}(\varphi)\right\|=K \geq$ 1 , where $K$ is the maximal dilatation $f$. Conversely, if $\varphi \in Q_{0}(R)$ satisfies the 
fundamental equation (1) (it is easy to see that $-\varphi$ also satisfies (1)), then either $\varphi$ or $-\varphi$ is associated with the Teichmüller mapping. Therefore the condition $\left\|g_{h}(\varphi)\right\| \geq 1$ will give us the right choice.

\section{ITERATIONS OF THE MAPPING BY HEIGHTS}

Let $R$ be a compact Riemann surface with punctures and let $g: R \rightarrow S$ be a quasiconformal mapping. The goal in this section is to define a sequence $\left\{\varphi_{n}\right\}_{1}^{\infty} \subset$ $Q_{0}(R)$, which will be proved to be a Hamilton sequence for the Teichmüller mapping $f$ in the homotopy class of $g$.

We shall first define a map $G: Q_{0}(R) \rightarrow Q_{0}(R)$ as follows. Let $\varphi_{1} \in Q_{0}(R)$ and let $\psi_{1}=g_{h}\left(\varphi_{1}\right) \in Q(S)$. We define $\tilde{\varphi}_{2}=\left(g_{h}\right)^{-1}\left(-\psi_{1}\right)$ and $\varphi_{2}=-\frac{\tilde{\varphi_{2}}}{\left\|\tilde{\varphi_{2}}\right\|} \in Q_{0}(R)$. At last we define $G\left(\varphi_{1}\right)=\varphi_{2}$.

According to the definition of mapping by heights, for any $c>0$ and $\varphi \in Q(R)$, we have $g_{h}(c \varphi)=c g_{h}(\varphi)$. From this and the definition of $G$ it is not hard to see that $G=-g_{\#}^{-1} \circ\left(-g_{\#}\right)$. This implies that $G$ is a homeomorphism from $Q_{0}(R)$ to $Q_{0}(R)$. Note that $\varphi$ is a fixpoint of $G$ if and only if $\varphi$ satisfies (1). In this sense, our main result below can be considered as a form of generalization of Theorem B.

For given $\varphi_{1} \in Q_{0}(R)$ and for each positive integer $n>1$, we define $\varphi_{n}=$ $G^{n-1}\left(\varphi_{1}\right)=G\left(\varphi_{n-1}\right)$. In this way, for each $\varphi_{1} \in Q_{0}(R)$, we can obtain a sequence of quadratic differentials $\left\{\varphi_{n}\right\}_{1}^{\infty} \subset Q_{0}(R)$.

Let $f$ be the Teichmüller mapping which is in the homotopy class of $g$ with the complex dilatation $\mu_{f}=k \frac{\bar{\varphi}_{0}}{\left|\varphi_{0}\right|}$, where $0 \leq k<1$ is a constant and $\varphi_{0} \in Q_{0}(R)$. Set $K=\frac{1+k}{1-k}$. Note that it is proved in [2] that

$$
\frac{1}{K} \leq\left\|g_{h}(\varphi)\right\| \leq K
$$

for any $\varphi \in Q_{0}(R)$.

The main result in this paper is the following.

Theorem 1. Let $g: R \rightarrow S$ be a quasiconformal mapping between a pair of compact or finitely punctured Riemann surfaces of hyperbolic type. Assume that $f$ is the Teichmüller mapping which is in the homotopy class of $g$ with complex dilatation $\mu_{f}=k \frac{\bar{\varphi}_{0}}{\left|\varphi_{0}\right|}$, where $0<k<1$ is a constant and $\varphi_{0} \in Q_{0}(R)$. Set $K=\frac{1+k}{1-k}$.

(i) If $\varphi_{1} \in Q_{0}(R)$ and $1 \leq\left\|g_{h}\left(\varphi_{1}\right)\right\|<K$, then $\left\{\varphi_{n}\right\}_{1}^{\infty}=\left\{G^{n-1}\left(\varphi_{1}\right)\right\}_{1}^{\infty}$ is a pairwise distinct sequence and $\varphi_{n} \rightarrow \varphi_{0}$ in norm as $n \rightarrow \infty$.

(ii) If $\varphi_{1} \in Q_{0}(R)$ and $\frac{1}{K}<\left\|g_{h}\left(\varphi_{1}\right)\right\|<1$, then $\left\{\varphi_{n}^{\prime}\right\}_{1}^{\infty}=\left\{G^{n-1}\left(-\varphi_{1}\right)\right\}_{1}^{\infty}$ is a pairwise distinct sequence and $\varphi_{n}^{\prime} \rightarrow \varphi_{0}$ in norm as $n \rightarrow \infty$.

(iii) If $\varphi_{1} \in Q_{0}(R)$ and $\left\|\psi_{1}\right\|=\left\|g_{h}\left(\varphi_{1}\right)\right\|=K$, then $\varphi_{1}$ is a fixpoint of $G$ and $\varphi_{1}=\varphi_{0}$. If $\varphi_{1} \in Q_{0}(R)$ and $\left\|\psi_{1}\right\|=\left\|g_{h}\left(\varphi_{1}\right)\right\|=\frac{1}{K}$, then $-\varphi_{1}$ is a fixpoint of $G$ and $-\varphi_{1}=\varphi_{0}$.

Corollary 2. Assume that $g$ and $f$ satisfy the assumptions of Theorem 1.

(i) If $\varphi_{1} \in Q_{0}(R)$ and $\left\|\psi_{1}\right\|=\left\|g_{h}\left(\varphi_{1}\right)\right\| \geq 1$, then $\left\{\varphi_{n}\right\}_{1}^{\infty}=\left\{G^{n-1}\left(\varphi_{1}\right)\right\}_{1}^{\infty}$ is a Hamilton sequence for $f$.

(ii) If $\varphi_{1} \in Q_{0}(R)$ and $\left\|\psi_{1}\right\|=\left\|g_{h}\left(\varphi_{1}\right)\right\|<1$, then $\left\{\varphi_{n}^{\prime}\right\}_{1}^{\infty}=\left\{G^{n-1}\left(-\varphi_{1}\right)\right\}_{1}^{\infty}$ is a Hamilton sequence for $f$. 


\section{Proof of Theorem 1}

Let $f$ be the Teichmüller mapping which is in the homotopy class of $g$ with the complex dilatation $\mu_{f}=k \frac{\bar{\varphi}_{0}}{\left|\varphi_{0}\right|}$, where $0<k<1$ is a constant and $\varphi_{0} \in Q_{0}(R)$. Set $K=\frac{1+k}{1-k}$.

We will first treat the case (i) and show that $\varphi_{n} \rightarrow \varphi_{0}$ in norm as $n \rightarrow \infty$.

To this aim, let $L_{n}=\left\|\psi_{n}\right\|$. We first prove that $\left\{L_{n}\right\}_{1}^{\infty}$ is an increasing sequence. Furthermore if $\varphi_{1} \neq \varphi_{0}$, then $\left\{L_{n}\right\}_{1}^{\infty}$ is a strictly increasing sequence.

Let $\tilde{\varphi}_{2}=\left(g_{h}\right)^{-1}\left(-\psi_{1}\right)$. We claim that $\left\|\tilde{\varphi}_{2}\right\| \geq L_{1}\left\|\psi_{1}\right\|$ and that if the equality holds, then $\varphi_{2}=\varphi_{1}=\varphi_{0}$.

To prove the claim, we denote a geometric local parameter of $R$ by $z=x+i y$, whereas, for $n \geq 1$, the parameter determined by $\varphi_{n}$ is

$$
z_{n}=x_{n}+i y_{n}=\int^{z} \sqrt{\varphi_{n}(z)} d z .
$$

The above specification is valid outside the zeros of $\varphi_{n}$. We have $\left|\varphi_{n}\right|=1 \mathrm{in}$ terms of the parameter. The parameter determined by $\tilde{\varphi}_{n}$ is

$$
\tilde{z}_{n}=\tilde{x}_{n}+i \tilde{y}_{n}=\int^{z} \sqrt{\tilde{\varphi}_{n}(z)} d z .
$$

It follows (from (29) in [3]) that

$$
\iint_{R}\left|\tilde{\varphi}_{2}(z)\right|^{\frac{1}{2}}\left|\varphi_{1}(z)\right|^{\frac{1}{2}} d x d y \geq L_{1}|| \varphi_{1} \|
$$

Applying the Schwarz inequality and dividing by $\left\|\varphi_{1}\right\|$, we obtain

$$
\left\|\tilde{\varphi}_{2}\right\| \geq L_{1}^{2}\left\|\varphi_{1}\right\|=L_{1}\left\|\psi_{1}\right\|
$$

If the inequality (2) becomes an equality, then (3) is also an equality. Therefore

$$
\begin{gathered}
L_{1}\left\|\varphi_{1}\right\|=\iint_{R}\left|\tilde{\varphi}_{2}\right|^{\frac{1}{2}} d x d y=\iint_{R} \sqrt{\left(\frac{\partial \tilde{x}_{2}}{\partial x_{1}}\right)^{2}+\left(\frac{\partial \tilde{y}_{2}}{\partial x_{1}}\right)^{2}} d x d y \\
\geq \iint_{R}\left|\frac{\partial \tilde{y}_{2}}{\partial x_{1}}\right| d x d y=L_{1}|| \varphi_{1}|| .
\end{gathered}
$$

This implies that $\frac{\partial \tilde{x}_{2}}{\partial x_{1}} \equiv 0$. Therefore we deduce that the trajectories of $\tilde{\varphi}_{2}$ are orthogonal to the trajectories of $\varphi_{1}$, and hence

$$
\tilde{\varphi}_{2}=\left(g_{h}\right)^{-1}\left(-\psi_{1}\right)=-L^{2} \varphi_{1} .
$$

Thus we have that

$$
g_{\#}\left(-\varphi_{1}\right)=-g_{\#}\left(\varphi_{1}\right) .
$$

Note that $\left\|g_{h}\left(\varphi_{1}\right)\right\| \geq 1$. It follows from Theorem B that $\varphi_{2}=\varphi_{1}=\varphi_{0}$. This proves our claim.

Next we set $\varphi_{2}=-\frac{\tilde{\varphi}_{2}}{\left\|\tilde{\varphi}_{2}\right\|}$ and $\psi_{2}=g_{h}\left(\varphi_{2}\right)$. We want to show $L_{2}=\left\|\psi_{2}\right\| \geq L_{1}=$ $\left\|\psi_{1}\right\|$ and the equality holds if and only if $\varphi_{1}=\varphi_{0}$.

Let $\left\|\tilde{\varphi}_{2}\right\|=\tilde{L}_{2}\left\|\psi_{1}\right\|$. Then we have proved that $\tilde{L}_{2} \geq L_{1}$. Now replacing $\varphi_{1}$ and $\psi_{1}$ by $\psi_{1}$ and $\tilde{\varphi}_{1}$ in the above argument and noting that

$$
\left\|\psi_{2}\right\|=\left\|g_{h}\left(\varphi_{2}\right)\right\|=L_{2}\left\|\varphi_{2}\right\|
$$


we deduce that $L_{2} \geq \tilde{L}_{2}$. Therefore

$$
\left\|\psi_{2}\right\|=L_{2} \geq \tilde{L}_{2} \geq L_{1}=\left\|\psi_{1}\right\| .
$$

If the equality $L_{2}=L_{1}$ holds, then we must have $\tilde{L}_{2}=L_{1}$. Consequently, we have $\varphi_{1}=\varphi_{0}$ again .

Repeating the above argument, we can prove that $L_{n} \leq L_{n+1}$ for all $n \geq 1$. If there is a $n_{0} \geq 1$ such that $L_{n_{0}}=L_{n_{0}+1}$, then we must have $\varphi_{0}=\varphi_{1}$.

Now let us recall three properties of the mapping by heights $g_{h}$. The first one is that

$$
\frac{1}{K} \leq \frac{\left\|g_{h}(\varphi)\right\|}{\|\varphi\|} \leq K
$$

for any $\varphi \in Q(R)$. The second one is that $\varphi \in Q_{0}(R)$ such that

$$
\left\|g_{h}(\varphi)\right\|=K
$$

if and only if $\varphi$ is the Teichmüller differential associated with the Teichmüller mapping $f$ in the homotopy class of $g$. The third one is that $g_{h}$ is a homeomorphism from $Q(R)$ to $Q(S)$. Refer to 2] and 3] for proofs of the first and second properties. The third property was clearly stated in [3] and, as it was noted by Strebel in [4, the proof of it follows from [2, Section 21.6].

It follows from the first property of $g_{h}$ that $L_{n} \leq K$ for all $n \geq 1$. Since $\left\|g_{h}\left(\varphi_{1}\right)\right\|=\left\|\psi_{1}\right\|<K$, it follows from the second property that $\varphi_{1} \not \equiv \varphi_{0}$. We deduce from the above argument that $L_{n}<K$ for all $n \geq 1$. This implies that $\left\{\varphi_{n}\right\}_{1}^{\infty}$ is a pairwise distinct sequence.

Now we are able to prove that $\varphi_{n} \rightarrow \varphi_{0}$ in norm as $n \rightarrow \infty$. Let

$$
K^{\prime}=\sup _{n}\left\{L_{n}\right\} \text {. }
$$

We first show that $K^{\prime}=K$. To prove this, take a subsequence $\left\{\varphi_{n_{j}}\right\}$ of $\left\{\varphi_{n}\right\}$ such that $\varphi_{n_{j}} \rightarrow \varphi_{0}^{\prime} \in Q_{0}(R)$ and $L_{n_{j}} \rightarrow K^{\prime}$ as $j \rightarrow \infty$. Using the property that $g_{h}: Q(R) \rightarrow Q(S)$ is continuous, we have

$$
\left\|g_{h}\left(\varphi_{0}^{\prime}\right)\right\|=K^{\prime}
$$

If $K^{\prime}<K$, using the property that $g_{h}: Q(R) \rightarrow Q(S)$ is a homeomorphism again, we must have $\left\|g_{h}\left(G\left(\varphi_{0}^{\prime}\right)\right)\right\|>K^{\prime}$. Therefore we would have $L_{n_{j}+1}>K^{\prime}$ for all sufficiently large $j$. This contradiction proves $K^{\prime}=K$. Therefore we deduce from the second property of the mapping by heights that $\varphi_{0}=\varphi_{0}^{\prime}$.

As $\left\{L_{n}\right\}_{1}^{\infty}$ is an increasing sequence which is convergent to $K$, any subsequence of $\left\{\varphi_{n}\right\}$ has a convergent subsequence which is convergent to $\varphi_{0}$. This implies that $\varphi_{n} \rightarrow \varphi_{0}$ as well. The proof of Case (i) is completed.

We next prove Case (ii).

Set $\psi_{1}=g_{\#}\left(\varphi_{1}\right)=\frac{g_{h}\left(\varphi_{1}\right)}{\left\|g_{h}\left(\varphi_{1}\right)\right\|} \in Q_{0}(S)$. Then we have $\left\|g_{h}^{-1}\left(\psi_{1}\right)\right\|>1$. Let $G_{1}=-g_{\#} \circ\left(-g_{\#}^{-1}\right)$ which is a homeomorphism from $Q_{0}(S)$ to $Q_{0}(S)$. Then Case (i) tells us $G_{1}^{n}\left(\psi_{1}\right) \rightarrow \psi_{0}$ in norm, as $n \rightarrow \infty$, where $\psi_{0}$ is the Teichmüller differential associated with $f^{-1}$. It follows from Theorem B that $g_{\#}^{-1}\left(-\psi_{0}\right)=-g_{\#}^{-1}\left(\psi_{0}\right)$. Let $\varphi_{0}^{\prime}=g_{\#}^{-1}\left(\psi_{0}\right)$. Then it is easy to check that $-\varphi_{0}^{\prime}$ satisfies $(1)$, that is, $g_{\#}\left(-\varphi_{0}^{\prime}\right)=$ $-g_{\#}\left(\varphi_{0}^{\prime}\right)$.

We next show $\left\|g_{h}\left(-\varphi_{0}^{\prime}\right)\right\|=K>1$. To this aim, let $\tilde{\varphi}_{0}^{\prime}=g_{h}^{-1}\left(\psi_{0}\right)$. Since $\psi_{0}$ is the Teichmüller quadratic differential for $f^{-1}$, it follows from the third property of the mapping by heights that $\left\|\tilde{\varphi}_{0}^{\prime}\right\|=K$. This also implies that $\varphi_{0}^{\prime}=\frac{\tilde{\varphi}_{0}^{\prime}}{K}$. Let 
$\tilde{\psi}_{0}=g_{h}\left(-\tilde{\varphi}_{0}^{\prime}\right)$. It is easy to see from the proof of the claim in Case (i) (also cf. (33) in [3] ) that

$$
\tilde{\psi}_{0}=g_{h}\left(-\tilde{\varphi}_{0}^{\prime}\right)=-K^{2} \psi_{0}
$$

Therefore

$$
\left\|g_{h}\left(-\varphi_{0}^{\prime}\right)\right\|=\frac{1}{K} K^{2}\left\|\psi_{0}\right\|=K>1
$$

Thus we deduce from Theorem B that $-\varphi_{0}^{\prime}=\varphi_{0}$, where $\varphi_{0}$ is the Teichmüller mapping associated with $f$.

Since $-\varphi_{0}^{\prime}=-g_{\#}^{-1}\left(\psi_{0}\right)$ and $-g_{\#}^{-1}$ is a homeomorphism from $Q_{0}(S) \rightarrow Q_{0}(S)$ and, for $n>1, G^{n}\left(-\varphi_{1}\right)=-g_{\#}^{-1} G_{1}^{n}\left(\psi_{1}\right)$, we deduce that $\left\{G^{n}\left(-\varphi_{1}\right)\right\}_{1}^{\infty}$ is a pairwise distinct sequence and $G^{n}\left(-\varphi_{1}\right) \rightarrow \varphi_{0}$ in norm, as $n \rightarrow \infty$. The proof of Case (ii) is completed.

We finally treat Case (iii).

Let $\varphi_{1} \in Q_{0}(R)$ and $\left\|\psi_{1}\right\|=\left\|g_{h}\left(\varphi_{1}\right)\right\|=K$. Then it follows from the second property of $g_{h}$ that $\varphi_{1}=\varphi_{0}$. It is easy to see from Theorem B and the definition of $G$ that $\varphi_{1}$ is a fixpoint of $G$.

Let $\varphi_{1} \in Q_{0}(R)$ and $\left\|\psi_{1}\right\|=\left\|g_{h}\left(\varphi_{1}\right)\right\|=\frac{1}{K}$. Then $-\varphi_{1}=\varphi_{0}$ and $-\varphi_{1}$ is a fixpoint of $G$.

The proof of Theorem 1 is completed.

\section{ACKNOWLEDGMENT}

The author would like to thank Kurt Strebel for his kind help during the preparation of the manuscript. He also wishes to thank the referee for his (her) useful suggestions.

\section{REFERENCES}

[1] F. Gardiner, Teichmüller theory and quadratic differentials, Wiley, New York, 1987. MR 88m:32044

[2] A. Marden and K. Strebel, The heights theorem for quadratic differentials on Riemann surfaces, Acta Math., 153(1984), 153-211. MR 86a:30076

[3] A. Marden and K. Strebel, A characterization of Teichmüller differentials, J. Differential Geometry, 37(1993), 1-29. MR 93m:32028

[4] K. Strebel, The elementary cases in Teichmüller mapping theorem, Ann. Acad. Sci. Fenn., Series A.I. Math., 15(1990), 319-328. MR 91m:30056

School of Mathematical Sciences, Peking University, Beijing 100871, People's RePUBLIC OF CHINA

E-mail address: wusj@pku.edu.cn 